\title{
Quasi-Hyperbolicity and Delay Semigroups
}

\author{
Shard Rastogi ${ }^{1}$ and Sachi Srivastava ${ }^{2}$ \\ ${ }^{1}$ Department of Mathematics, University of Delhi, Delhi 110 007, India \\ ${ }^{2}$ Department of Mathematics, University of Delhi, South Campus, Benito Juarez Road, New Delhi 110 021, India
}

Correspondence should be addressed to Shard Rastogi; sharadrastogi2@gmail.com

Received 1 June 2016; Accepted 28 September 2016

Academic Editor: Jozef Banas

Copyright (C) 2016 S. Rastogi and S. Srivastava. This is an open access article distributed under the Creative Commons Attribution License, which permits unrestricted use, distribution, and reproduction in any medium, provided the original work is properly cited.

We study quasi-hyperbolicity of the delay semigroup associated with the equation $u^{\prime}(t)=B u(t)+\Phi u_{t}$, where $u_{t}$ is the history function and $(B, D(B))$ is the generator of a quasi-hyperbolic semigroup. We give conditions under which the associated solution semigroup of this equation generates a quasi-hyperbolic semigroup.

\section{Introduction}

We consider the abstract delay differential equation:

$$
(\mathrm{DE})\left\{\begin{array}{l}
u^{\prime}(t)=B u(t)+\Phi u_{t}, \quad t \geqslant 0, \\
u(0)=x_{0} \\
u_{0}=f_{0}
\end{array}\right.
$$

where $X$ is a Banach space, $u_{t}(\theta)=u(t+\theta),-1 \leq \theta \leq$ $0, x_{0} \in X, f$ lies in an appropriate space, and we assume that $(B, D(B))$ generates a $C_{0}$-semigroup on $X$ and that $\Phi$ : $W^{1, p}([-1,0], X) \rightarrow X$, the delay operator, is bounded and linear. One approach to the study of the abstract theory of such equations is via semigroups of operators, pioneered by Hale [1], Webb [2], and Kraskovi, among others. This involves associating a $C_{0}$-semigroup (defined on an appropriate state space) with the above delay equation, and whose orbits correspond to the mild solutions of (DE). Analysis of qualitative and asymptotic behaviour of this semigroup then yields detailed information about the solutions of the delay equation (see [3-6] for more details).

In this paper we study the property of quasi-hyperbolicity of the semigroup associated with the delay equation (DE). Recently, Batty and Tomilov [5] have introduced and studied quasi-hyperbolicity of $C_{0}$-semigroups, motivated by questions arising in the context of quasi-Anosov diffeomorphisms. Precisely, a $C_{0}$-semigroup $(T(t))_{t \geqslant 0}$ is quasi-hyperbolic if there exists $t>0$ (independent of $x$ ) such that $\max (\|T(2 t) x\|$, $\|x\|) \geq 2\|T(t) x\|$ for all $x \in X$. They show in particular that quasi-hyperbolicity of a $C_{0}$-semigroup is closely connected to the generator satisfying certain lower bounds. Quasi-hyperbolicity of a $C_{0}$-semigroup generalises the property of hyperbolicity of $C_{0}$-semigroup. Hyperbolicity of the delay semigroup has been explained in detail in $[3,4]$, and we follow their approach here. In particular we prove that if $(B, D(B))$ generates a quasi-hyperbolic semigroup, and the delay operator is small in some sense, then the associated delay semigroup remains quasi-hyperbolic. This is the main result, proved in Sections 3 and 4, for Hilbert spaces and Banach spaces, respectively. Section 2 is of a preliminary nature containing known fact about delay equation and delay semigroups. Throughout this paper, we follow the notations of [3].

\section{Preliminaries}

We collect here some basic results about delay differential equations that will be required in the sequel and refer to [3], for details. Throughout this paper, we shall consider the equation (DE), where $p \in[1, \infty)$ is fixed and

(1) $x_{0} \in X$ is a Banach space,

(2) $B: D(B) \subseteq X \rightarrow X$ is the generator of a $C_{0}$-semigroup,

(3) $f_{0} \in L^{p}([-1,0], X)$, 
(4) $\Phi: W^{1, p}([-1,0], X) \rightarrow X$ is a linear, bounded operator,

(5) $u:[-1, \infty) \rightarrow X$ and $t \geqslant 0$, and $u_{t}:[-1,0] \rightarrow X$ is defined by $u_{t}(\sigma):=u(t+\sigma), \sigma \in[-1,0]$.

Let $\mathbf{X}=X \times L^{p}([-1,0], X)$ and define the operator $\mathscr{A}$ on $\mathbf{X}$ as follows:

$$
\mathscr{A}:=\left(\begin{array}{cc}
B & \Phi \\
0 & A_{0}
\end{array}\right)
$$

where

$$
\begin{aligned}
& D(\mathscr{A}) \\
& :=\left\{\left(\begin{array}{l}
x \\
f
\end{array}\right) \in D(B) \times W^{1, p}([-1.0], X): f(0)=x\right\},
\end{aligned}
$$

$A_{0}$ is the generator of the left shift semigroup $\left(T_{0}(t)\right)_{t \geqslant 0}$ on $L^{p}([-1,0], X)$. Thus, $D\left(A_{0}\right)=\left\{f \in W^{1, p}([-1,0], X): f(0)=\right.$ $0\}, A_{0} f=f^{\prime}$ and

$$
\left(T_{0}(t) f\right)(s):= \begin{cases}f(t+s) & \text { if } s \in[-1,0], t+s \leq 0 \\ 0 & \text { if } s \in[-1,0], t+s>0 .\end{cases}
$$

The following relations between the solutions of the abstract Cauchy problem

$$
(\mathrm{ACP})\left\{\begin{array}{l}
v^{\prime}(t)=\mathscr{A} v(t) \quad t \geqslant 0, \\
v(0)=v_{0},
\end{array}\right.
$$

associated with the operator matrix $(\mathscr{A}, D(\mathscr{A}))$ on the Banach space $\mathbf{X}$ with initial value $v_{0}=\left(\begin{array}{c}x_{0} \\ f_{0}\end{array}\right)$ and (DE) are well known [3, Lemma 2.2]:

(i) If $u$ is a solution of (DE), then $t \mapsto\left(\begin{array}{c}u(t) \\ u_{t}\end{array}\right)$ is a solution of the equation (ACP).

(ii) If $t \mapsto\left(\begin{array}{c}u(t) \\ v(t)\end{array}\right)$ is a solution of (ACP), then $v(t)=u_{t}$ for all $t \geqslant 0$ and $u$ is a solution of (DE).

Further (DE) is well posed if and only if (ACP) is well posed if and only if $(\mathscr{A}, D(\mathscr{A}))$ generates a strongly continuous $\operatorname{semigroup}(\mathscr{T}(t))_{t \geqslant 0}$ on $\mathbf{X}=X \times L^{p}([-1,0], X)$. In the case that (DE) is well posed, the semigroup $(\mathscr{T}(t))_{t \geqslant 0}$ is called the delay semigroup corresponding to (DE). Then the solutions (both mild and classical) of (DE) are determined by the semigroup $(\mathscr{T}(t))_{t \geqslant 0}$. We shall be exploring the properties of the delay semigroup $(\mathscr{T}(t))_{t \geqslant 0}$, associated with (DE).

We note here that since in this paper we shall be working with $p \in[1, \infty)$, fixed, we write (DE) instead of (DE) ${ }_{p},(\mathrm{ACP})$ instead of $(\mathrm{ACP})_{p}$ and so on, the dependence on $p$ being implicit.

\section{Quasi Hyperbolic Semigroups and Delay Equations}

The delay operator $\Phi \in \mathscr{L}\left(W^{1, p}([-1,0], X), X\right)$ is said to be admissible (see [3]) if the operator $(\mathscr{A}, D(\mathscr{A}))$ is the generator of a strongly continuous semigroup on $\mathbf{X}=X \times L^{p}([-1,0], X)$ for each generator $(B, D(B))$ on $X$ and the function $\lambda \mapsto$ $\Phi R\left(\lambda, A_{0}\right)$ is a bounded analytic function on the half plane $\{\lambda \in \mathbb{C}: \operatorname{Re} \lambda>\omega\}$ for all $\omega \in R$.

A $C_{0}$-semigroup $(T(t))_{t \geqslant 0}$ is said to be quasi-hyperbolic [5, Definition 3.1] if there exists $t>0$ (independent of $x$ ) such that

$$
\max (\|T(2 t) x\|,\|x\|) \geq 2\|T(t) x\| \quad \forall x \in X .
$$

For $C_{0}$-semigroups defined on Hilbert spaces, the following complete characterisation of such a semigroup is available in terms of the generator.

Theorem 1 (see [5, Corollary 3.10]). Let $B$ be the generator of a $C_{0}$-semigroup $(T(t))_{t \geqslant 0}$ on a Hilbert space $H$. Then $(T(t))_{t \geqslant 0}$ is quasi-hyperbolic if and only if

$$
\|(B-i s) x\| \geq c\|x\|, \quad \forall x \in D(B)
$$

for some $c>0$.

The following result gives conditions under which the delay semigroup is quasi-hyperbolic.

Theorem 2. Let $H$ be a Hilbert space and consider the delay equation $(D E)$ with $p=2$. Assume that $\Phi$ is admissible, and the semigroup $(B, D(B))$ generates a quasi-hyperbolic $C_{0^{-}}$ semigroup. If the strict inequality

$$
\sup _{s \in \mathbb{R}}\left\|\Phi R\left(i s, A_{0}\right)\right\|<1
$$

holds, then $(\mathscr{A}, D(\mathscr{A}))$ generates a quasi-hyperbolic $C_{0}$-semigroup.

Proof. Let $(B, D(B))$ generate a quasi-hyperbolic semigroup. Therefore, there exists $c>0$ such that

$$
\|(B-i s) x\| \geq c\|x\|, \quad x \in D(B) .
$$

As $\Phi$ is admissible, $(\mathscr{A}, D(\mathscr{A}))$ generates a $C_{0}$-semigroup on $H \times L^{2}([-1,0], H)$. From (8), for every $f \in D\left(A_{0}\right)$, we have

$$
\|\Phi f\|_{H} \leq m\left\|\left(A_{0}-i s\right) f\right\|_{L^{2}([-1,0], H)},
$$

where $m$ is a constant strictly less than 1 . Moreover, the semigroup generated by $\left(A_{0}, D\left(A_{0}\right)\right)$ on the Hilbert space $L^{2}([-1,0], \mathrm{H})$ is hyperbolic, hence quasi hyperbolic. Therefore, it follows that $\left\|\left(A_{0}-i s\right) f\right\| \geq m_{2}\|f\|$ for all $f \in D\left(A_{0}\right)$. 
Now for $\left(\begin{array}{l}x \\ f\end{array}\right) \in D(\mathscr{A}), s \in \mathbb{R}$, and above $m$, we have, on using (9) and (10),

$$
\begin{aligned}
\left\|(\mathscr{A}-i s)\left(\begin{array}{c}
x \\
f
\end{array}\right)\right\|_{H \times L^{2}([-1,0], H)} \\
=\left\|\left(\begin{array}{cc}
(B-i s) & \Phi \\
0 & \left(A_{0}-i s\right)
\end{array}\right)\left(\begin{array}{l}
x \\
f
\end{array}\right)\right\|_{H \times L^{2}([-1,0], H)} \\
=\|(B-i s) x+\Phi f\|_{H}+\left\|\left(A_{0}-i s\right) f\right\|_{L^{2}([-1,0], H)} \\
\geq\|(B-i s) x\|_{H}-\|\Phi f\|_{H}+\left\|\left(A_{0}-i s\right) f\right\|_{L^{2}([-1,0], H)} \\
\geq c\|x\|_{H}-\|\Phi f\|_{H}+\left\|\left(A_{0}-i s\right) f\right\|_{L^{2}([-1,0], H)} \\
\geq c\|x\|_{H}-m\left\|\left(A_{0}-i s\right) f\right\|_{L^{2}([-1,0], H)} \\
\quad+\left\|\left(A_{0}-i s\right) f\right\|_{L^{2}([-1,0], H)} \\
\geq c^{\prime}\left(\|x\|_{H}+\|f\|_{L^{2}([-1,0], H)}\right) .
\end{aligned}
$$

Thus, for all $s \in \mathbb{R}$, and $\left(\begin{array}{l}x \\ f\end{array}\right) \in D(\mathscr{A})$,

$$
\left\|(\mathscr{A}-i s)\left(\begin{array}{c}
x \\
f
\end{array}\right)\right\|_{H \times L^{2}([-1,0], H)} \geq c^{\prime}\left\|\left(\begin{array}{l}
x \\
f
\end{array}\right)\right\|_{H \times L^{2}([-1,0], H)} .
$$

It follows from Theorem 1 that $(\mathscr{A}, D(\mathscr{A}))$ generates a quasihyperbolic $C_{0}$-semigroup.

Example 3. Let $\eta$ be a function of bounded variation on $[-1,0]$ such that $|\eta|([-1,0]) \leq k$, for $k<1$, and $\Phi: C([-1,0]$, $X) \rightarrow X$ be given by the Riemann-Stieltjes integral

$$
\Phi(f)=\int_{-1}^{0} f d \eta
$$

for all $f \in C([-1,0], X)$. For $p=2$, $\Phi$ satisfies the conditions of Theorem 2. It has been shown in [3] that $\Phi$ extends to a bounded admissible delay operator from $W^{1, p}([-1,0], X)$ to $X$.

Since $A_{0}$ generates a nilpotent semigroup, $\sigma\left(A_{0}\right) \cap i \mathbb{R}$ is empty. Moreover, for $f \in L^{2}([-1,0], X)$

$$
\begin{aligned}
& \left\|\Phi R\left(i s, A_{0}\right) f\right\|=\left\|\int_{-1}^{0} d \eta(\sigma)\left(R\left(i s, A_{0}\right) f\right)(\sigma)\right\| \\
& =\left\|\int_{-1}^{0} d \eta(\sigma) \int_{0}^{\infty} e^{-i s t}\left(T_{0}(t) f\right)(\sigma) d t\right\| \\
& =\left\|\int_{-1}^{0} d \eta(\sigma) \int_{\sigma}^{0} e^{i s(\sigma-r)} f(r) d r\right\| \\
& \leq \int_{-1}^{0} \int_{\sigma}^{0}\|f(r)\| d r d|\eta|(\sigma) \\
& \leq \int_{-1}^{0} \int_{-1}^{0}\|f(r)\| d r d|\eta|(\sigma) \leq\|f\|_{2} \int_{-1}^{0} d|\eta|(\sigma) \\
& \quad=|\eta|([-1,0])\|f\|_{2} .
\end{aligned}
$$

So if we choose $|\eta|([-1,0]) \leq k$, then such a $\Phi$ would satisfy

$$
\sup _{s \in \mathbb{R}}\left\|\Phi R\left(i s, A_{0}\right)\right\|<1 .
$$

Hence, by Theorem $2,(\mathscr{A}, D(\mathscr{A}))$ generates quasi-hyperbolic $C_{0}$-semigroup.

The following example adapted from [5, Example 3.14] illustrates Theorem 2.

Example 4. Define $w: \mathbb{R} \rightarrow \mathbb{R}$, as

$$
w(s)= \begin{cases}e^{-s}, & s \leq 0 \\ e^{s}, & \text { otherwise }\end{cases}
$$

Then $w$ is a strictly positive, continuous function, satisfying

$$
\begin{aligned}
& \text { (i) } \frac{w(s)}{w(s-t)} \leq c e^{\omega t}, \\
& \text { (ii) } \lim _{n \rightarrow+\infty} \frac{w(n)}{w(n-1)}=e, \\
& \text { (iii) } \lim _{n \rightarrow-\infty} \frac{w(n)}{w(n-1)}=\frac{1}{e}
\end{aligned}
$$

for some $c, \omega \in \mathbb{R}$. Then

$$
\lim _{n \rightarrow \pm \infty} \frac{w(n)}{w(n-1)}=w_{ \pm}, \quad w_{-}<1<w_{+} .
$$

Let weighted right shift $C_{0}$-semigroup $(T(t))_{t \geqslant 0}$ on $L_{2}(\mathbb{R})$ be given by

$$
(T(t) f)(s)=\frac{w(s)}{w(s-t)} f(s-t)
$$

Then $(T(t))_{t \geqslant 0}$ is a $C_{0}$-semigroup on $L_{2}(\mathbb{R})$ with generator say, $B$. By [5, Example 3.14], $(B, D(B))$ generates a quasi-hyperbolic $C_{0}$-semigroup, which is not hyperbolic. Let $\Phi$ be as in Example 3. Now, by our Theorem 2 it follows that the delay semigroup $(\mathscr{T}(t))_{t \geqslant 0}$, associated with (DE)

$$
\begin{aligned}
u^{\prime}(t) & =B u(t)+\Phi u_{t}, \quad t \geqslant 0, \\
u(0) & =x_{0}, \\
u_{0} & =f_{0}
\end{aligned}
$$

is quasi-hyperbolic.

Remark 5. It follows from proof of Theorem 2 that lower bounds for $B$ imply lower bounds for the delay semigroup generator $\mathscr{A}$, even on general Banach spaces, provided condition (8) holds. Precisely if $\|(B-i s) x\| \geq c\|x\|, c>0 \forall x \in D(B)$ and (8) holds, then

$$
\left\|(\mathscr{A}-i s)\left(\begin{array}{l}
x \\
f
\end{array}\right)\right\| \geq c\left\|\left(\begin{array}{l}
x \\
f
\end{array}\right)\right\|, \quad c>0,\left(\begin{array}{l}
x \\
f
\end{array}\right) \in D(\mathscr{A}) .
$$




\section{Banach Space Case}

In this section we obtain conditions for quasi-hyperbolicity of the delay semigroup associated with (DE) when the underlying space is a general Banach space. Consider (DE) with $p \in[1, \infty)$ fixed. Let $B$ be a densely defined, closed linear operator on a Banach space $X$, and $S(\mathbb{R}, X)$ denote the space of $X$-valued Schwartz function. For $\varphi \in \mathcal{S}(\mathbb{R})$ and $x \in D(B)$, we write $(\varphi \otimes x)(t)=\varphi(t) x,(t \in \mathbb{R})$. Let $S_{B}(\mathbb{R}, X)=\operatorname{lin}\{\varphi \otimes$ $x: x \in D(B), \varphi \in \mathcal{S}(\mathbb{R})\}$ and $\mathscr{F}$ be the Fourier transform on $S(\mathbb{R}, X)$. Note that $S_{B}(\mathbb{R}, X)$ is dense in $L_{p}(\mathbb{R}, X)$ for every $p \in$ $[1,+\infty)$. Further, since $A_{0}$ generates a hyperbolic semigroup [7, Theorem 2.7], it follows from [4] that $R\left(i \cdot, A_{0}\right)$ is an $L^{1}$ Fourier multiplier from $L^{1}\left(\mathbb{R}, L^{1}([-1,0], X)\right)$ into itself; that is, for $Y=L^{p}([-1,0], X)$ the operator $\mathbf{M}_{0}: S(\mathbb{R}, Y) \rightarrow$ $S(\mathbb{R}, Y)$, given by

$$
\mathbf{M}_{0}(f)=\mathscr{F}^{-1}\left(R\left(i \cdot A_{0}\right) \mathscr{F} f\right),
$$

extends to a bounded linear operator on $L^{p}(\mathbb{R}, Y)$. We recall from [5] the definition of a lower $L_{p}$ Fourier multiplier.

Definition 6. The linear operator

$$
\begin{aligned}
M_{B-i \cdot}: S_{B}(\mathbb{R}, X) & \longmapsto S(\mathbb{R}, X), \\
\left(M_{B-i} f\right)(s) & =(B-i s) f(s),
\end{aligned}
$$

is said to be a lower $L_{p}(\mathbb{R}, X)$-Fourier multiplier if there exists $c>0$ such that

$$
\left\|\mathscr{F}^{-1} M_{B-i} \cdot \mathscr{F} f\right\|_{L_{p}} \geq c\|f\|_{L_{p}}
$$

for every $f \in S_{B}(\mathbb{R}, X)$.

Note that

$$
\left(\mathscr{F}^{-1} M_{B-i} \cdot \mathscr{F} f\right)(s)=B f(s)-f^{\prime}(s) .
$$

Batty and Tomilov [5] have given the following characterisation for quasi-hyperbolic in terms of lower Fourier multipliers.

Theorem 7 (see [5, Theorem 3.9]). Let $(T(t))_{t \geqslant 0}$ be a $C_{0^{-}}$ semigroup on a Banach space $X$ with generator $B$. Then $(T(t))_{t \geqslant 0}$ is quasi-hyperbolic if and only if $M_{B-i}$. is a lower $L_{p}(\mathbb{R}, X)$-Fourier multiplier for all/some $p \in[1,+\infty)$.

We are now able to deduce quasi-hyperbolicity of the delay semigroup on $X \times L^{p}([-1,0], X)$ under the assumption of quasi-hyperbolicity of the semigroup generated by $B$, under certain conditions.

Theorem 8. Let $X$ be a Banach space and consider the delay equation $(D E)$, with $p \in[1, \infty)$ fixed. Assume that the delay $\Phi$ and the operator $B$ satisfy the following conditions:

(1) $(\mathscr{A}, D(\mathscr{A}))$ is a generator of a $C_{0}$-semigroup on $Z=$ $X \times L^{p}([-1,0], X)$ whenever $\left(B, D(B)\right.$ generates a $C_{0^{-}}$ semigroup on $X$.
(2) $\Phi R\left(i \cdot, A_{0}\right)$ is a $L^{p}$ Fourier multiplier for some/all $p \in$ $[1, \infty)$ from $L^{p}(\mathbb{R}, Y)$ to $L^{p}(\mathbb{R}, X)$; that is, the map $\widetilde{M}_{p}: S(\mathbb{R}, Y) \rightarrow S(\mathbb{R}, X)$, given by

$$
\widetilde{M}_{p} f:=\mathscr{F}^{-1}\left(\Phi R\left(i \cdot A_{0}\right) \mathscr{F} f\right),
$$

extends to a bounded linear operator from $L^{p}(\mathbb{R}, Y)$ to $L^{p}(\mathbb{R}, X)$,

(3) $\left\|\widetilde{M}_{1}\right\|_{\mathscr{L}\left(L^{1}(\mathbb{R}, Y), L^{1}(\mathbb{R}, X)\right)}<1$.

(4) the semigroup generated by $(B, D(B))$ is quasihyperbolic.

Then $(\mathscr{A}, D(\mathscr{A}))$ generates a quasi-hyperbolic $C_{0}$-semigroup.

Proof. Let $(B, D(B))$ generate a quasi-hyperbolic semigroup. Then, by Theorem 7 , for $p=1$, there exists $c>0$ such that, for $\Phi \in \mathscr{L}\left(W^{1, p}([-1,0], X), X\right)$,

$$
\begin{aligned}
& \left\|\mathscr{F}^{-1} M_{B-i} \cdot \mathscr{F}(\varphi \otimes x)\right\|_{L^{1}(\mathbb{R}, X)} \\
& \quad=\left\|B(\varphi \otimes x)-\varphi^{\prime} \otimes x\right\|_{L^{1}(\mathbb{R}, X)} \geq c\|\varphi \otimes x\|_{L^{1}(\mathbb{R}, X)} .
\end{aligned}
$$

We show that $M_{\mathscr{A}-i}$. is a lower $L_{1}(\mathbb{R}, Z)$-Fourier multiplier. Observe that since $M_{B-i}$. maps $S_{B}(\mathbb{R}, X) \mapsto S(\mathbb{R}, X), M_{\mathscr{A}-i}$. maps $S_{\mathscr{A}}(\mathbb{R}, Z) \mapsto S(\mathbb{R}, Z)$. Recall that $\left(A_{0}, D\left(A_{0}\right)\right)$ is the generator of the nilpotent left shift semigroup $\left(T_{0}(t)\right)_{t \geqslant 0}$ in $L^{p}\left(\mathbb{R}, L^{p}([-1,0], X)\right)$.

Since $\Phi R\left(i \cdot A_{0}\right)$ is an $L^{1}(\mathbb{R}, Y)$ Fourier multiplier, therefore, there exists $0<m_{1}<1$ such that, for all $g \in L^{1}(\mathbb{R}, Y)$,

$$
\left\|\widetilde{M}_{1}(g)\right\|_{L^{1}(\mathbb{R}, X)} \leq m_{1}\|g\|_{L^{1}(\mathbb{R}, Y)} .
$$

In particular, for $f \in D\left(A_{0}\right)$ and $\varphi \in S(\mathbb{R})$, letting $g=$ $\left(\mathscr{F}^{-1} M_{A_{0}-i} \mathscr{F}\right)(\varphi \otimes f)=\varphi \otimes\left(A_{0} f\right)-\varphi^{\prime} \otimes f \in L^{1}(\mathbb{R}, Y)$, it follows from (28) that for all $\varphi \in S(\mathbb{R}), f \in D\left(A_{0}\right)$

$$
\begin{aligned}
& \|\Phi(\varphi \otimes f)\|_{L^{1}(\mathbb{R}, X)} \\
& \quad \leq m_{1}\left\|\left(\mathscr{F}^{-1} M_{A_{0}-i} \cdot \mathscr{F}\right)(\varphi \otimes f)\right\|_{L^{1}(\mathbb{R}, Y)} \\
& \quad \leq m_{1}\left\|A_{0}(\varphi \otimes f)-\left(\varphi^{\prime} \otimes f\right)\right\|_{L^{1}(\mathbb{R}, Y)} .
\end{aligned}
$$

Now, for $Z=X \times L^{1}([-1,0], X)$, we have, on using (27) and (29), that

$$
\begin{aligned}
& \left\|\mathscr{F}^{-1} M_{\mathscr{A}-i} \mathscr{F}\left(\varphi \otimes\left(\begin{array}{c}
x \\
f
\end{array}\right)\right)\right\|_{L^{1}(\mathbb{R}, Z)} \\
& =\left\|\left(\begin{array}{cc}
B & \Phi \\
0 & A_{0}
\end{array}\right)\left(\begin{array}{c}
\varphi \otimes x \\
\varphi \otimes f
\end{array}\right)-\left(\begin{array}{c}
\varphi^{\prime} \otimes x \\
\varphi^{\prime} \otimes f
\end{array}\right)\right\|_{L^{1}(\mathbb{R}, Z)} \\
& =\left\|\left(\begin{array}{c}
B(\varphi \otimes x)+\Phi(\varphi \otimes f)-\varphi^{\prime} \otimes x \\
A_{0}(\varphi \otimes f)-\varphi^{\prime} \otimes f
\end{array}\right)\right\|_{L^{1}(\mathbb{R}, X) \times L^{1}\left(\mathbb{R}, L^{1}([-1,0], X)\right)}
\end{aligned}
$$




$$
\begin{aligned}
= & \left\|B(\varphi \otimes x)+\Phi(\varphi \otimes f)-\varphi^{\prime} \otimes x\right\|_{L^{1}(\mathbb{R}, X)} \\
& +\left\|A_{0}(\varphi \otimes f)-\varphi^{\prime} \otimes f\right\|_{L^{1}\left(\mathbb{R}, L^{1}([-1,0], X)\right)} \\
\geq & \left\|B(\varphi \otimes x)-\varphi^{\prime} \otimes x\right\|_{L^{1}(\mathbb{R}, X)}-\|\Phi(\varphi \otimes f)\|_{L^{1}(\mathbb{R}, X)} \\
& +\left\|A_{0}(\varphi \otimes f)-\varphi^{\prime} \otimes f\right\|_{L^{1}\left(\mathbb{R}, L^{1}([-1,0], X)\right)} \\
\geq & c\|\varphi \otimes x\|_{L^{1}(\mathbb{R}, X)} \\
& +\left(1-m_{1}\right)\left\|A_{0}(\varphi \otimes f)-\varphi^{\prime} \otimes f\right\|_{L^{1}\left(\mathbb{R}, L^{1}([-1,0], X)\right)} \\
= & c\|\varphi \otimes x\|_{L^{1}(\mathbb{R}, X)}+c^{\prime}\|\varphi \otimes f\|_{L^{1}\left(\mathbb{R}, L^{1}([-1,0], X)\right)},
\end{aligned}
$$

where we have used the fact that $A_{0}$ is the generator of a hyperbolic semigroup and therefore also generator of quasi hyperbolic semigroup so that $A_{0}-i$. is a lower $L^{1}$ Fourier multiplier. Thus,

$$
\begin{aligned}
& \left\|\mathscr{F}^{-1} M_{\mathscr{A}-i} \mathscr{F}\left(\varphi \otimes\left(\begin{array}{c}
x \\
f
\end{array}\right)\right)\right\|_{L^{1}(\mathbb{R}, Z)} \\
& \geq c_{1}\left(\|\varphi \otimes x\|_{L^{1}(\mathbb{R}, X)}+\|\varphi \otimes f\|_{L^{1}\left(\mathbb{R}, L^{1}([-1,0], X)\right)}\right), \\
& \left\|\mathscr{F}^{-1} M_{\mathscr{A}-i} \cdot \mathscr{F}\left(\varphi \otimes\left(\begin{array}{c}
x \\
f
\end{array}\right)\right)\right\|_{L^{1}(\mathbb{R}, Z)} \\
& \geq \mathcal{c}_{1}\left\|\varphi \otimes\left(\begin{array}{c}
x \\
f
\end{array}\right)\right\|_{L^{1}(\mathbb{R}, Z)} .
\end{aligned}
$$

It follows similarly that

$$
\left\|\mathscr{F}^{-1} M_{\mathscr{A}-i} \cdot \mathscr{F} g\right\|_{L^{1}(\mathbb{R}, Z)} \geq c_{1}\|g\|_{L^{1}(\mathbb{R}, Z)}
$$

for every $g \in S_{\mathscr{A}}(\mathbb{R}, Z)=\operatorname{lin}\left\{\varphi \otimes\left(\begin{array}{l}x \\ f\end{array}\right):\left(\begin{array}{l}x \\ f\end{array}\right) \in D(\mathscr{A}), \varphi \in\right.$ $\delta(\mathbb{R})\}$. It now follows from Theorem 7 that $(\mathscr{A}, D(\mathscr{A}))$ generates a quasi-hyperbolic $C_{0}$-semigroup.

We next turn to $L^{p}(\mathbb{T}, X)$ multipliers. Following Batty and Tomilov [5], we set

$$
\begin{aligned}
& P(\mathbb{T}, X):=\left\{\sum_{k=-n}^{n} \xi^{k} x_{k}: \xi \in \mathbb{T}, x_{k} \in X, n \in \mathbb{N}\right\}, \\
& P_{B}(\mathbb{T}, X):=\left\{\sum_{k=-n}^{n} \xi^{k} x_{k}: \xi \in \mathbb{T}, x_{k} \in D(B), n \in \mathbb{N}\right\},
\end{aligned}
$$

and say that the linear operator $M_{B-i}: P_{B}(\mathbb{T}, X) \mapsto P(\mathbb{T}, X)$, defined by

$$
M_{B-i}\left(\sum_{k=-n}^{n} \xi^{k} x_{k}\right):=\sum_{k=-n}^{n} \xi^{k}(B-i k) x_{k},
$$

is a lower $L_{p}(\mathbb{T}, X)$-Fourier multiplier if there exists $c>0$ such that

$$
\left\|\sum_{k=-n}^{n} \xi^{k}(B-i k) x_{k}\right\|_{L^{p}(\mathbb{T}, X)} \geq c\left\|\sum_{k=-n}^{n} \xi^{k} x_{k}\right\|_{L^{p}(\mathbb{T}, X)}
$$

for every $n \in \mathbb{N}, x_{k} \in D(B)$. Recall from [5] that $R\left(i \cdot A_{0}\right)$ is an $L^{p}\left(\mathbb{T}, L^{p}([-1,0], X)\right)$ Fourier multiplier for $p \in[1, \infty)$. The following theorem relates the approximate spectrum of an operator from the delay semigroup to that of the semigroup generated by $(B, D(B))$.

Theorem 9. Let $X$ be a Banach space and consider the delay equation (DE). Assume that $\Phi$ satisfies the following conditions:

(1) $(\mathscr{A}, D(\mathscr{A}))$ is a generator of a $C_{0}$-semigroup on $Z=$ $X \times L^{p}([-1,0], X)$ whenever $(B, D(B))$ generates a $C_{0}$ semigroup on $X$.

(2) $\Phi R\left(i \cdot, A_{0}\right)$ is a $L^{p}$ Fourier multiplier for some/all $p \in$ $[1, \infty)$ from $L^{p}(\mathbb{T}, Y)$ to $L^{p}(\mathbb{T}, X)$, that is, the map $M_{p}$ : $P(\mathbb{T}, Y) \rightarrow P(\mathbb{T}, X)$ given by

$$
M_{p}\left(\sum_{k=-n}^{n} \xi^{k} x_{k}\right):=\sum_{k=-n}^{n} \xi^{k} \Phi R\left(i k, A_{0}\right) x_{k}
$$

extends to a bounded linear operator from $L^{p}(\mathbb{T}, Y)$ to $L^{p}(\mathbb{T}, X)$,

(3) $\left\|M_{1}\right\|_{\mathscr{L}\left(L^{1}(\mathbb{T}, Y), L^{1}(\mathbb{T}, X)\right)}<1$.

Let $(T(t))_{t \geqslant 0}$ be $C_{0}$-semigroup generated by the operator $B$ and $(\mathscr{T}(t))_{t \geqslant 0}$ be the delay semigroup generated by $(\mathscr{A}, D(\mathscr{A}))$ on $X \times L^{p}([-1,0], X), p \in[1, \infty)$ fixed. Then $1 \notin \sigma_{a p}(T(2 \pi))$ implies $1 \notin \sigma_{a p}(\mathscr{T}(2 \pi))$.

Proof. Suppose $1 \notin \sigma_{a p}(T(2 \pi))$. Then by [5, Theorem 3.12], $M_{B-i}$. is a lower $L_{p}(\mathbb{T}, X)$ Fourier multiplier for $p=1$, and there exists $c>0$ such that

$$
\left\|\sum_{k=-n}^{n} \xi^{k}(B-i k) x_{k}\right\|_{L^{1}(\mathbb{T}, X)} \geq c\left\|\sum_{k=-n}^{n} \xi^{k} x_{k}\right\|_{L^{1}(\mathbb{T}, X)}
$$

for every $n \in \mathbb{N}, x_{k} \in D(B)$. We show that $M_{\mathscr{A}-i}$. is lower $L_{p}(\mathbb{T}, Z)$-Fourier multiplier. Note that $M_{\mathscr{A}-i}: P_{\mathscr{A}}(\mathbb{T}, Z) \mapsto$ $P(\mathbb{T}, Z)$. Since $\Phi R\left(i \cdot, A_{0}\right)$ is an $L^{1}\left(\mathbb{T}, L^{1}([-1,0], X)\right)$ Fourier multiplier, therefore, there exists $0<m_{1}<1$ and for all $n \in \mathbb{N}$, $g_{k} \in L^{1}\left(\mathbb{T}, L^{1}([-1,0], X)\right)$

$$
\begin{aligned}
& \left\|M_{1}\left(\sum_{k=-n}^{n} \xi^{k} g_{k}\right)\right\|_{L^{1}(\mathbb{T}, X)} \\
& \leq m_{1}\left\|\sum_{k=-n}^{n} \xi^{k} g_{k}\right\|_{L^{1}\left(\mathbb{T}, L^{1}([-1,0], X)\right)} .
\end{aligned}
$$

Choosing $g_{k}=\left(i k-A_{0}\right) f_{k}, f_{k} \in D\left(A_{0}\right)$, in the above inequality, we get

$$
\begin{aligned}
& \left\|\Phi\left(\sum_{k=-n}^{n} \xi^{k} f_{k}\right)\right\|_{L^{1}(\mathbb{T}, X)} \\
& \quad \leq m_{1}\left\|\sum_{k=-n}^{n} \xi^{k}\left(A_{0}-i k\right) f_{k}\right\|_{L^{1}\left(\mathbb{T}, L^{1}([-1,0], X)\right)} .
\end{aligned}
$$


Now, for $Z=X \times L^{1}([-1,0], X)$, we have, for $x_{k} \in D(B)$, $f_{k} \in D\left(A_{0}\right)$, on using (37) and (39), that

$$
\begin{aligned}
& \left\|\sum_{k=-n}^{n} \xi^{k}(\mathscr{A}-i k)\left(\begin{array}{c}
x_{k} \\
f_{k}
\end{array}\right)\right\|_{L^{1}(\mathbb{T}, Z)} \\
& =\left\|\sum_{k=-n}^{n}\left(\begin{array}{c}
\xi^{k}(B-i k) x_{k}+\xi^{k} \Phi f_{k} \\
\xi^{k}\left(A_{0}-i k\right) f_{k}
\end{array}\right)\right\|_{L^{1}(\mathbb{T}, Z)} \\
& =\left\|\sum_{k=-n}^{n} \xi^{k}(B-i k) x_{k}+\sum_{k=-n}^{n} \xi^{k} \Phi f_{k}\right\|_{L^{1}(\mathbb{T}, X)} \\
& +\left\|\sum_{k=-n}^{n} \xi^{k}\left(A_{0}-i k\right) f_{k}\right\|_{L^{1}\left(\mathbb{T}, L^{1}([-1,0], X)\right)} \\
& \geq\left\|\sum_{k=-n}^{n} \xi^{k}(B-i k) x_{k}\right\|_{L^{1}(\mathbb{T}, X)}-\left\|\sum_{k=-n}^{n} \xi^{k} \Phi f_{k}\right\|_{L^{1}(\mathbb{T}, X)} \\
& +\left\|\sum_{k=-n}^{n} \xi^{k}\left(A_{0}-i k\right) f_{k}\right\|_{L^{1}\left(\mathbb{\pi}, L^{1}([-1,0], X)\right)} \\
& \geq c\left\|\sum_{k=-n}^{n} \xi^{k} x_{k}\right\|_{L^{1}(\mathbb{T}, X)} \\
& +\left(1-m_{1}\right)\left\|\sum_{k=-n}^{n} \xi^{k}\left(A_{0}-i k\right) f_{k}\right\|_{L^{1}\left(\mathbb{T}, L^{1}([-1,0], X)\right)} \\
& =c\left\|\sum_{k=-n}^{n} \xi^{k} x_{k}\right\|_{L^{1}(\mathbb{T}, X)} \\
& +c^{\prime}\left\|\sum_{k=-n}^{n} \xi^{k} f_{k}\right\|_{L^{1}\left(\mathbb{T}, L^{1}([-1,0], X)\right)},
\end{aligned}
$$

where we have used the fact that $A_{0}-i$. is a lower $L^{1}$ Fourier multiplier. Thus,

$$
\begin{gathered}
\left\|\sum_{k=-n}^{n} \xi^{k}(\mathscr{A}-i k)\left(\begin{array}{c}
x_{k} \\
f_{k}
\end{array}\right)\right\|_{L^{1}(\mathbb{T}, Z)} \\
\geq c\left\|\sum_{k=-n}^{n} \xi^{k}\left(\begin{array}{c}
x_{k} \\
f_{k}
\end{array}\right)\right\|_{L^{1}(\mathbb{T}, Z)}
\end{gathered}
$$

for some $c>0$ and $\left(\begin{array}{l}x_{k} \\ f_{k}\end{array}\right) \in D(\mathscr{A})$. It follows from [5, Theorem 3.12] that $1 \notin \sigma_{a p}(\mathscr{T}(2 \pi))$.

\section{Competing Interests}

The authors declare that they have no competing interests.

\section{Acknowledgments}

The first author would like to thank CSIR for JRF Fellowship while the second author acknowledges with gratitude the support from the R \& D grant of University of Delhi.

\section{References}

[1] J. K. Hale, Functional Differential Equation, vol. 3 of Applied Mathematical Sciences, Springer, New York, NY, USA, 1971.

[2] G. F. Webb, "Functional differential equations and nonlinear semigroups in $L^{P}$-spaces," Journal of Differential Equations, vol. 20, no. 1, pp. 71-89, 1976.

[3] A. Bátkai, "Hyperbolicity of linear partial differential equations with delay," Integral Equations and Operator Theory, vol. 44, no. 4, pp. 383-396, 2002.

[4] A. Batkai, E. Fasanga, and R. Shvidkoy, "Hyperbolicity of delay equations via Fourier multipliers," Acta Scientiarum Mathematicarum, vol. 69, no. 1-2, pp. 131-145, 2003.

[5] C. J. K. Batty and Y. Tomilov, "Quasi-hyperbolic semigroups," Journal of Functional Analysis, vol. 258, no. 11, pp. 3855-3878, 2010.

[6] C. J. Batty, "Differentiability and growth bounds of solutions of delay equations," Journal of Mathematical Analysis and Applications, vol. 299, no. 1, pp. 133-146, 2004.

[7] Y. Latushkin and R. Shvidkoy, "Hyperbolicity of semigroups and fourier multipliers," in Systems, Approximation, Singular Integral Operators, and Related Topics (Bordeaux, 2000), vol. 129 of Operator Theory: Advances and Applications, pp. 341-363, Birkhäuser, Basel, Switzerland, 2000. 


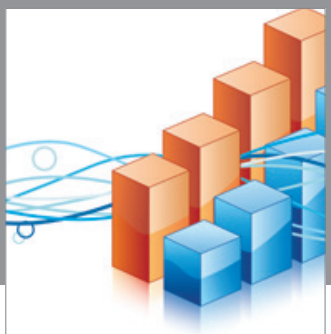

Advances in

Operations Research

vatem alat4

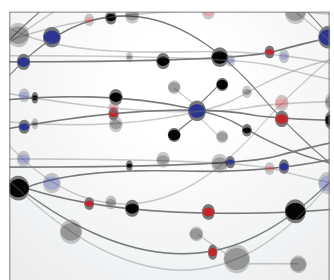

\section{The Scientific} World Journal
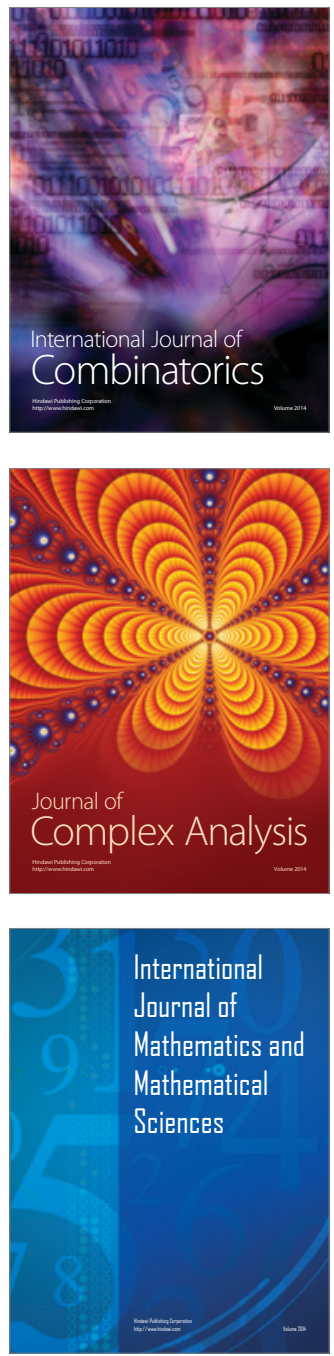
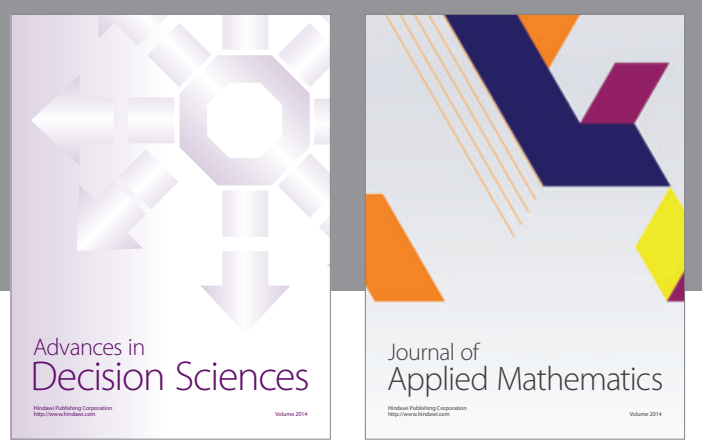

Algebra

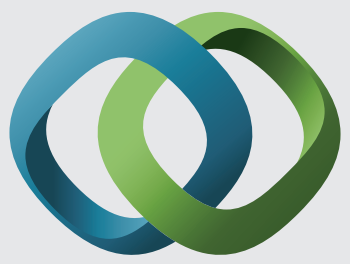

\section{Hindawi}

Submit your manuscripts at

http://www.hindawi.com
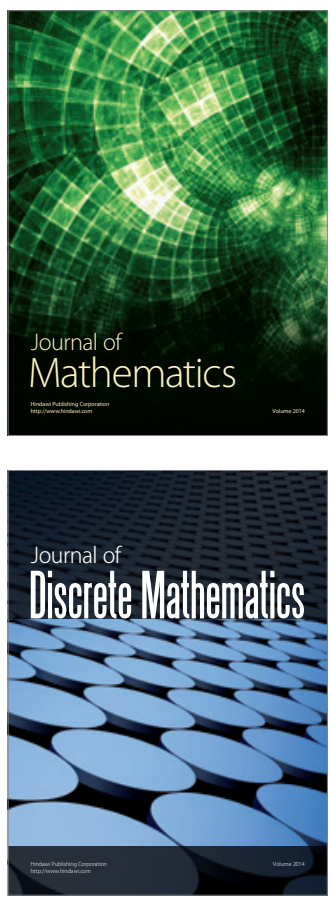

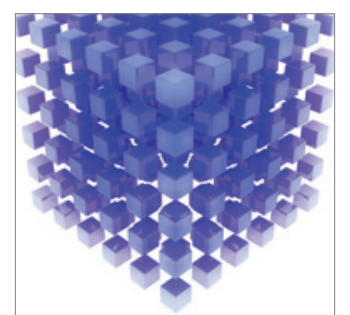

Mathematical Problems in Engineering
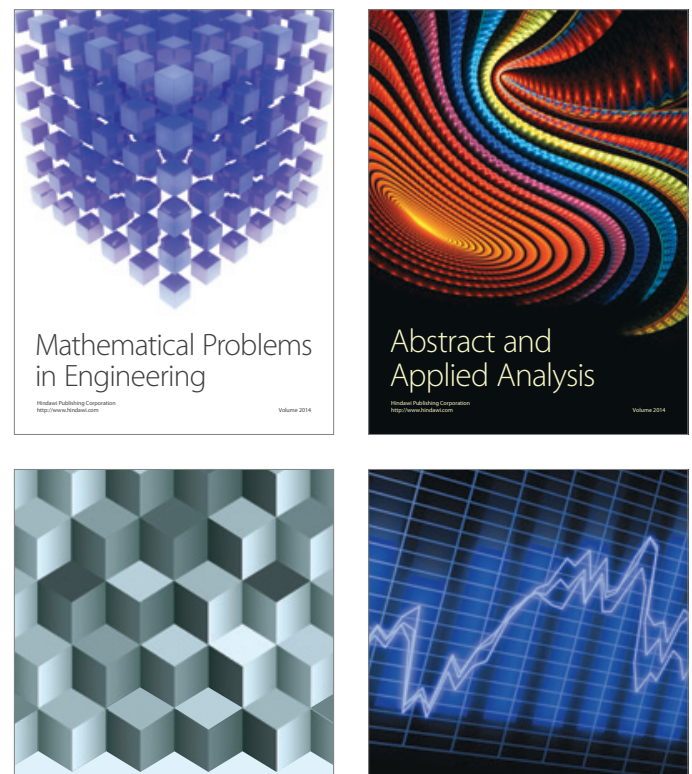

Journal of

Function Spaces

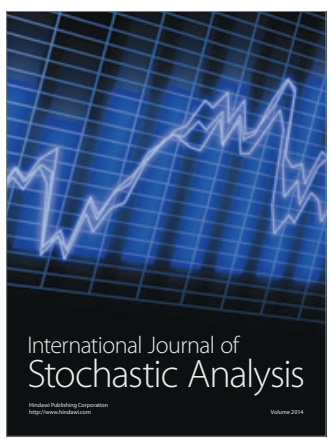

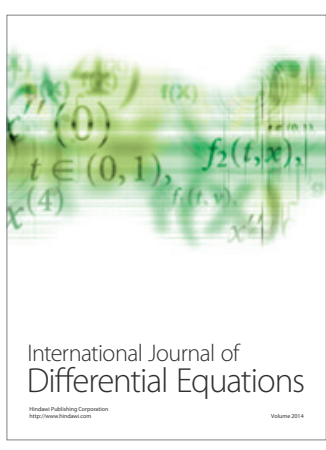
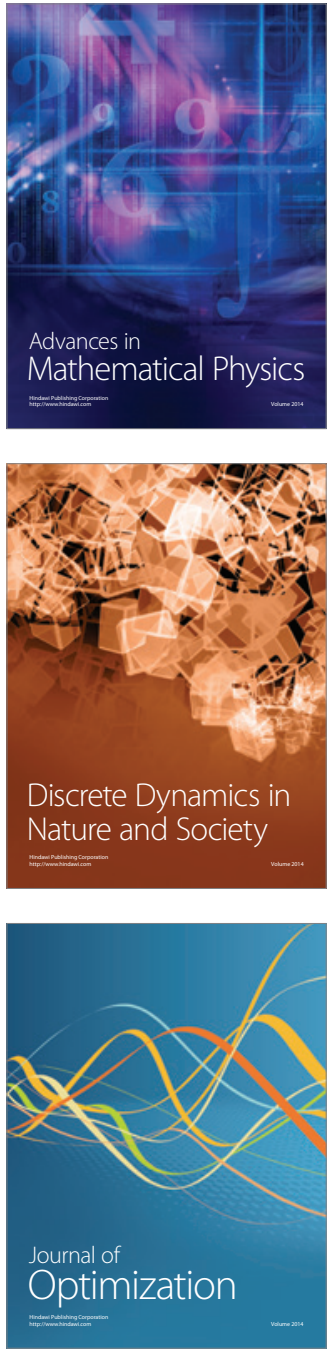